\title{
A importância das Tecnologias da Informação e Comunicação em cursos online: Um estudo exploratório em uma Universidade do Sul de Minas Gerais
}

\author{
Lílian FERRUGINI ${ }^{1}$ \\ Rodrigo Cassimiro FREITAS ${ }^{2}$ \\ Donizeti Leandro SOUZA ${ }^{3}$ \\ Cleber Carvalho CASTRO ${ }^{4}$
}

\begin{abstract}
${ }^{1}$ Graduada em Administração (UFJF), especialista em MBA em Finanças (UFJF), atualmente é mestranda em Administração (UFLA).

${ }^{2}$ Graduado em Administração (PUC-MG), Pesquisador do Grupo de Estudos em Redes, Estratégia e Inovação GEREI/UFLA. atualmente é mestrando em Administração (UFLA). ${ }^{3}$ Graduado em Administração de empresas (UNIVAS), especialista em Gestão de pessoas (UNIFEI) e em Gestão executiva de negócios (PUC-MINAS), atualmente é mestrando em Administração (UFLA). ${ }^{4}$ Graduado em Administração (UFLA), Mestrado em Administração (UFRGS) e Doutorado em Agronegócio (UFRGS), atualmente é professor adjunto na UFLA e coordenador institucional da UAB/UFLA.
\end{abstract}

Recebido em: 20/05/2013 - Aprovado em: 30/07/2013 - Disponibilizado em: 15/08/2013

\begin{abstract}
RESUMO
Tendências do mundo atual giram em torno de manter-se atualizado e conectado às diversas e rápidas informações que surgem a todo instante nos mais diversos lugares. As Tecnologias da Informação e Comunicação (TIC's), dessa forma, possuem o objetivo de facilitar a transmissão de informações e promover a criação de novos conhecimentos com eficácia, proporcionando o encurtamento das distâncias geográficas. O presente estudo tem como objetivo demonstrar, por meio de uma pesquisa qualitativa, de que forma as TIC's são utilizadas no curso online para formação de tutores que atuarão em um curso de Administração Pública à Distância que atuarão em um curso de Administração Pública à Distância de uma grande universidade federal do sul de Minas Gerais e, como elas podem contribuir para a criação e difusão do conhecimento entre os cursistas. Verificou-se que os indivíduos, a partir da disponibilização de ferramentas como, fóruns de discussão, wiki, chat, e-mails, entre outras ferramentas, criam possibilidades rápidas e dinâmicas de compartilhamento de transferência de conhecimento. Identifica-se que as TIC's, são elementos essenciais e fundamentais para a modalidade de Educação a Distância, visto essas serem ferramentas que permitem a disponibilização de informações e conhecimentos a distintos e diversos lugares e pessoas simultaneamente.

PALAVRAS CHAVE: Educação a Distância, Curso online, tutor, TIC's, Conhecimento.

\section{The importance of Information Technology and Communication in online courses: An exploratory study at a south of University of Minas Gerais}

\begin{abstract}
Tendencies of today's world revolve around keeping up to date and connected to diverse and fast information that arise all the time in many different places. The Information and Communication Technologies (ICTs) thus have the purpose of facilitating the transmission of information and promote the creation of new knowledge, providing shortening of geographical distances. This study aims to demonstrate, through a qualitative study how ICTs are used in the online course for training tutors who will act toward a degree in Public Administration Distance of a big federal southern university Minas Gerais and how they can contribute to the creation and diffusion of knowledge among course participants. It was verified that individuals from the availability of tools such as discussion forums, wiki, chat, e-mails, among other tools, create possibilities quick dynamic sharing of knowledge transfer. Identified that ICTs are essential and fundamental elements to the form of distance education, as these are tools that enable the provision of information and knowledge and many different places and people simultaneously.
\end{abstract}

KEY WORDS: Distance Education, Online Course, tutor, ICT's, Knowledge. 


\section{1 - INTRODUÇÃO}

A Educação a Distância (EaD) tem o objetivo de proporcionar capacitação, qualificação e geração de novos conhecimentos para os indivíduos. Para isso, um dos fatores essenciais e necessário é a formação do corpo docente (professores/tutores) que irão atuar diretamente nesta modalidade de educação. Esses devem ser preparados para executarem adequadamente suas funções de forma a promover o sucesso do curso e o efetivo aprendizado dos alunos. Assim, o corpo docente primeiramente necessita de especialização e cursos de capacitação que promovam o aprendizado necessário para o desempenho de suas atividades.

Nesse sentido, surgem os cursos para formação de tutores, os quais têm a finalidade de orientar os tutores que mediarão o processo de aprendizagem, visto ser de extrema importância o domínio do conteúdo e da metodologia utilizada nesse modelo de educação.

Para auxiliar na disseminação e eficiência dos cursos supracitados, utilizam-se algumas ferramentas moderadoras do conhecimento. Nesse contexto, julga-se que as TIC's (Tecnologia da Informação e Comunicação) possibilitariam maior compartilhamento e cocriação de conhecimento entre os cursistas.
As TIC's são instrumentos necessários e indispensáveis, tanto para os cursistas quanto para os tutores, perante a necessidade de disseminação do conhecimento e da formação de novas competências frente à escassez de tempo e do fluxo intenso de informações enfrentado pelos indivíduos e organizações.

Nesse escopo de atuação, o presente trabalho tem como objetivo principal demonstrar de que forma as TIC's são utilizadas no curso online para formação de tutores que atuarão em um curso de Administração Pública à Distância de uma grande universidade federal do sul de Minas Gerais e como elas podem contribuir para a criação e difusão do conhecimento entre os cursistas. Diante disso, pretende-se compreender como os tutores se relacionam com essas ferramentas e a importância das mesmas no processo de capacitação.

\section{2 - REFERÊNCIAL TEÓRICO}

\subsection{Peculiaridades da Educação à Distância (EaD).}

A EaD existe há várias décadas e teve seu início com a colaboração de correspondências via correios, telégrafos, fax, entre outros. Nos anos de 1990 com a expansão e disseminação da internet, o processo de aprendizado à distância tornou-se mais fácil, rápido e eficiente com a ampliação 
da inovação e a disponibilidade de tecnologias para auxílio à transmissão de dados e informações (BORBA; MALHEIROS; ZULATTO, 2008).

Neste contexto, devido à crescente exigência do mercado de trabalho, se torna crucial a obtenção de informações relevantes, conhecimento, educação, desenvolvimento de habilidades e qualificação profissional, a $\mathrm{EaD}$ têm promovido e se consolidado como uma opção para aqueles que não dispõem de tempo suficiente para se dedicar à realização de seus estudos, considerando-se para tanto os aspectos da modalidade presencial (OLIVEIRA, 2010; MACHADO et al., 2011).

O principal objetivo da $\mathrm{EaD}$ é superar barreiras reduzindo fronteiras que impedem o acesso à informação e ao conhecimento. O espaço geográfico não é mais um agravante que impossibilita o aprendizado contínuo. A $\mathrm{EaD}$ tem a finalidade de proporcionar o encurtamento de distâncias com a possibilidade de gerenciamento eficiente do tempo por parte daqueles que optam por essa modalidade de educação. Nesse sentido, Pretti (2000) elucida algumas vantagens da EaD tais como: alcance geográfico, estudo em tempo livre, oportunidade de interação, comunicação assíncrona.

De acordo com Pompeu e Silva Filho (2005), a EaD surge a partir da combinação de ferramentas tecnológicas inovadoras que permitem que os sujeitos estudem individualmente ou em grupos, através do auxílio de métodos orientados à distância.

Nesse contexto, a evolução e a propagação da internet configuraram como peças-chave para disseminação da $\mathrm{EaD}$ em locais onde não há condições favoráveis para alcance do modelo presencial de educação.

Azevedo et al. (2011) advogam que o aprendizado e a formação de cada profissional é um processo continuado, visto que os mesmos necessitam aprender e dominar com qualidade novas tecnologias para se manterem atualizados em relação às diferentes demandas que surgem no mercado hiperdinâmico.

Devido às constantes mudanças no contexto contemporâneo ocasionadas em partes pelo advento da inovação e da tecnologia é de extrema importância relatar que os profissionais que irão atuar como mediadores da modalidade de $\mathrm{EaD}$ enfrentarão muitos desafios, na medida em que são eles os responsáveis por dinamizar o processo de ensino/aprendizagem. Acredita-se que esses profissionais provavelmente irão enfrentar muitas barreiras vindas dos próprios alunos (dificuldades com uso de tecnologia, com acesso a internet, conhecimento, disciplina, etc.), da própria habilidade em utilizar recursos novos, dinâmicos e tecnológicos, assim como na forma específica 
de interação e construção do conhecimento em si (COELHO et al., 2011).

Assim sendo, os mediadores (tutores/professores) de cursos à distância devem considerar todos os obstáculos e dificuldades a fim de buscarem alternativas que possam facilitar e desenvolver desempenho eficaz do aprendizado, ao passo que os indivíduos consigam melhorar suas qualidades perante o mercado globalizado.

\subsection{TIC's aplicadas à EaD}

Com a rápida, crescente e globalizada informatização do mundo atual, as TIC's tornaram-se ferramentas de apoio e de disseminação de vieses capazes de viabilizar o crescimento e dinamizar a EaD. A utilização de recursos de Tecnologia de Informação e Comunicação no escopo da $\mathrm{EaD}$ torna-se fonte imediata de informação e conhecimento ao delinear o tempo e o espaço como não mais impedidores de criação de conhecimentos. Nesse sentido, Nogueira (1996) enfatiza que as TIC's possibilitam a redução de distâncias ao mesmo tempo em que dão margem à disseminação do conhecimento.

\section{Corroborando estes conceitos,} Mendonça et al. (2011); Finch e Jacobs (2012); Rienties, Brouwer e Lygo-Baker (2013) advogam que as novas TIC's, adotadas sistematicamente no meio educacional, proporcionam relações mais ousadas na medida que o tempo e o espaço assumem proporções mais reduzidas. Assim, tem-se que tempo e espaço não podem ser mais questionados como obstáculos para a disseminação de informações.

Há de se destacar que as TIC's estão presentes, indispensavelmente, nos processos educacionais, seja em cursos de graduação, pós-graduação e de capacitação (AZEVEDO, 2001), proporcionando interação entre os indivíduos, em um processo de aprendizado interativo.

As TIC's utilizadas na EaD integram e viabilizam-se através de uma plataforma específica no Ambiente Virtual de Aprendizagem (AVA), sendo composta por ferramentas desenvolvidas de auxílio à interação dos cursistas, como por exemplo os fóruns de discussão, tarefas, chats, wikis, vídeos, glossário, questionários, podcast, entre outras. Essas ferramentas possuem a função de desenvolver recursos educacionais responsáveis pela disseminação de informações e da possibilidade de contato e troca de conhecimento entre seus participantes, além de contribuir para a transformação do aprendizado (ABREU; GONÇALVES; PRAGNOZZI, 2003).

Assim, as TIC's servem como instrumentos para aperfeiçoar o processo de formação dos indivíduos, sendo consideradas como facetas que intermediam a comunicação e o compartilhamento de informações entre os 
alunos e os tutores/professores, os quais, em sua maioria, estão separados temporal e espacialmente (BARRETO, 2003; ABREU; GONÇALVES; PRAGNOZZI, 2003).

Do mesmo modo, Pretto e Riccio (2010), enfatizam que, tanto os discentes quanto os docentes, com auxílio das TIC's, adquirem a qualidade de desenvolver e potencializar sua autonomia e pensamento crítico, deixando de lado as informações e os aprendizados massificados, ou seja, deixam de ser meramente consumidores de informação para serem criadores de uma nova cultura a novas ideias e formas de pensar e desenvolver habilidades.

Nessa perspectiva, identificar como se relacionam as TIC's que irão auxiliar na formação dos profissionais que atuarão em cursos a distância torna-se crucial para se entender como esses futuros profissionais poderão desenvolver suas estratégias de ensino na disseminação e criação de novos conhecimentos exigidos pelo mercado. Nesse sentido, diante das principais funções do tutor, defende-se que,

“[...] ele precisa organizar, administrar e regular situações de aprendizagem, deixando de ser o "dono do saber" e o controlador da aprendizagem, para tornar-se um mediador que estimula a curiosidade, o debate e a interação com os outros participantes do processo" (SENO; BELHOT, 2009. p. 503).
Marek (2009) também descreve que os profissionais que irão lecionar na modalidade à distância devem receber treinamento específico, visto ser uma forma peculiar e diferente do modelo tradicional (presencial), em que os profissionais atuantes precisam desenvolver habilidades pedagógicas para lecionarem eficientemente.

\section{3 - OBJETO DE ESTUDO: CURSO ONLINE PARA TUTORES EM UMA UNIVERSIDADE FEDERAL MINEIRA}

Com o avanço da internet, as propostas de curso online tornam-se atrativas e de fácil aceitação pelos indivíduos, dada sua capacidade de difusão de informação e de conhecimento possibilitados por ferramentas disponíveis em rede, o que permite a comunicação em tempo real ou não (BORBA; MALHEIROS; ZULATTO, 2008).

$\mathrm{O}$ curso online para tutores que atuarão no curso de Administração Pública a distância ministrado em uma universidade federal mineira consistiu em duas etapas: um curso presencial com duração de sete horas e meia em que foram descritas as principais funções do tutor e a inserção dos primeiros contatos com a plataforma moodle de ensino, e posteriormente, um curso online, em que os tutores tiveram a oportunidade de expor suas características básicas, como formação educacional, cidade de origem, atividades que gosta de desenvolver, como também os 
objetivos almejados ao atuarem como tutores de um curso a distância.

Ao longo do curso foram expostas questões/questionamentos que cotidianamente os tutores irão se deparar. As questões tinham o objetivo de divulgar as condutas e os principais questionamentos dos alunos. Assim sendo, o objetivo era criar discussões e interações entre os tutores para escolherem o melhor caminho para a solução de respostas e mediação online. A partir dessas interações, os tutores, com intermediação de um coordenador/mediador, puderam compartilhar suas experiências e ao mesmo tempo desenvolverem respostas eficientes para os questionamentos expostos. Assim, os tutores puderam trocar informação, experiências (para aqueles que já atuaram anteriormente como tutores), relatos e impressões sobre as atividades a serem desenvolvidas.

Os cursantes puderam ainda contar com atividades diárias que envolveram perguntas e respostas diretas e de forma assíncrona, fóruns de discussão sobre determinada temática abordada, dúvidas e mediação de discussões a respeito das principais atividades desenvolvidas ao longo do curso. Também foram desenvolvidos chats entre grupos formados pelos participantes de forma síncrona. Assim como explanado por Alipour, Karimi e Idris (2011) a transferência e criação de conhecimento se dá entre os membros da organização a partir da interação e da troca de suas experiências, ideias e perspectivas.

\section{4 - METODOLOGIA}

Para atingir os objetivos pretendidos utilizou-se a estratégia de pesquisa qualitativa descritiva-exploratória (VERGARA, 2005). Ressalta-se que a estratégia qualitativa engloba diversas técnicas com a finalidade de explorar, descrever e codificar a simbologia de um emaranhado de dados significantes para a pesquisa (BANDEIRA-DE-MELO E CUNHA, 2003).

A exploração do material se deu ao passo de organização e categorização para implementação desses procedimentos. Assim, foram realizadas entrevistas (via e-mail) através de questionários semiestruturados com 11 questões discursivas.

$\mathrm{O}$ presente curso contou com a presença de 29 participantes. Os questionários foram enviados para todos os participantes, configurando-se uma amostragem nãoprobabilística por conveniência. Neste tipo de amostragem os indivíduos são escolhidos simplesmente por serem mais acessíveis ou então por serem mais fáceis de serem avaliados (ALENCAR, 2000; BRUNI, 2010).

Contudo, responderam ao questionário em torno de $70 \%$ dos participantes (21 respondentes), índice de resposta considerado alto para pesquisas feitas por meio eletrônico 
(COOPER; SCHINDLER, 2003; MALHOTRA, 2006).

As perguntas realizadas foram baseadas no propósito central de identificar de que forma as TIC's são utilizadas e como elas podem contribuir para a criação e difusão do conhecimento entre os participantes do curso online.

Algumas conversas informais também foram realizadas entre os pesquisadores e alguns participantes a fim de coletar diretamente percepções e impressões a cerca da participação no respectivo curso.

Por fim, o tratamento e a interpretação referiram-se à geração de inferências e dos resultados da investigação. A técnica de tratamento dos dados escolhida e apropriada para esse trabalho foi a análise de conteúdo proposta por Bardin (1995), pois buscou-se atingir maior descrição no conteúdo das mensagens dos entrevistados.

Dessa forma pôde-se investigar e concluir com maior exatidão a importância e a eficácia das TIC's na difusão do conhecimento entre os participantes do respectivo curso.

\section{5 - RESULTADOS E DISCUSSÃO}

A partir da análise dos dados foram identificadas algumas observações interessantes a respeito da utilização das TIC's no processo de aprendizado e disseminação do conhecimento entre os cursistas.

Ao discutir a primeira pergunta em que foi questionada qual a primeira impressão com o curso online, os entrevistados demonstraram expectativa positiva em relação ao curso e que o mesmo seria simples. Não obstante, disseram haver muito conteúdo e pouca interação do mediador do curso com os participantes. Esse fato demonstra claramente a necessidade de interação intensiva entre o mediador do curso e seus participantes para o sucesso de qualquer curso online, fato extremamente importante em cursos à distância.

Entre as perguntas 2, 3 e 4 foram questionados a importância do curso, os aspectos positivos e o que mais agradou pelo curso ter sido online. Dentre as respostas coletadas verificou-se que o que mais agradou os participantes foi a flexibilidade de tempo para participar das atividades, assim como a capacidade de pensar e recorrer a outros materiais para postar as respostas e consertar os erros de forma simples e dinâmica. Para os que vão atuar como futuros mediadores de disciplinas de $\mathrm{EaD}$, o curso é visto como de extrema importância por abordar um primeiro contato com a plataforma de ensino e para aprender a dinâmica de um curso à distância.

Tais relatos demonstram as particularidades e as diferenças de um curso na modalidade à distância em detrimento do 
presencial, possuindo aspectos distintos que devem ser reconhecidos antes de se aderir ao mesmo, visto haver extrema flexibilidade para realização das tarefas. Entretanto é imprescindível disciplina, comprometimento e responsabilidade dos cursistas.

Em relação às perguntas 5 e 6 foram questionados se a inexistência do curso online, assim como das TIC's, proporcionaria a mesma aprendizagem. Os respondentes relataram que a troca de informações no curso ministrado online foi muito mais proveitosa e eficiente que no curso presencial realizado na semana anterior. Um entrevistado relatou a seguinte frase em relação ao curso online. “... a absorção do conhecimento de forma interativa lhe permite enxergar determinados contextos de formas diferentes a partir da visão do outro" (X3). No mesmo sentido, todos afirmaram ser impossível, na atualidade, aprendizagem sem o uso dos recursos e ferramentas das TIC's, visto essas terem a função de simplificar e tornar mais ágeis e dinâmicos os processos de troca de informação e conhecimento. Outros entrevistados também relataram que sem o curso online, não seria possível o primeiro contato com a plataforma moodle, visto muitos não terem atuado ainda como tutores de curso a distância.

Contudo, alguns respondentes disseram que poderiam ter sido exploradas mais questões e exigida uma maior interação entre os membros para que os mesmos se conhecessem melhor. Esse fato demonstra que o tutor, ao mediar a interação com os discentes, deve sempre exigir a participação dos mesmos, pois os discentes muitas vezes postam suas considerações e posteriormente não interagem mais nas discussões.

Já as questões 7 e 8 versaram sobre a importância das TIC's em sua formação educacional e se as TIC's utilizadas no curso online atenderam as necessidades. Nesse contexto, foram feitas várias assertivas a cerca da utilização das TIC's. (X4) relata que as TIC's “... facilita a aprendizagem, agiliza a formação e o encontro de conhecimentos, possibilita os relacionamentos pessoais e as trocas de informações mais ágeis". Percebese então a indispensabilidade das TIC's para o processo de trocas de informações e conhecimento e, consequentemente, de aprendizado.

Alguns entrevistados também relataram que mesmo em cursos na modalidade presencial as TIC's são fatores fundamentais para auxiliar na troca de informação e conhecimento. A maioria concordam que as TIC's utilizadas no respectivo curso online atenderam às necessidades para a realização do curso. Outros já disseram que não reconheciam a sistemática dos recursos de TIC's, assim como não imaginavam a existência de um 
conjunto complexo de ferramentas que possibilitam a visualização de todas as atividades realizadas pelos cursistas.

Apresentando as últimas questões 9, 10 e 11, as quais abordaram questionamentos sobre a utilização das TIC's via computador e a consequente transferência de conhecimentos entre os participantes. A maioria dos respondentes afirmaram que as TIC's facilitam a troca de informações entre os membros do grupo de discussão através de ferramentas como: fóruns, chats, wikis, etc. Contudo, não houve espaço, tempo e exigência suficientes para a disseminação de informações. Percebe-se, nesse sentido, que as TIC's são propulsoras indispensáveis para criação e difusão do conhecimento. Entretanto os indivíduos ainda necessitam de abordagem e exigência contínua por parte dos mediadores, ou seja, os tutores e professores.

\section{6 - CONSIDERAÇOES FINAIS}

A trajetória do estudo demonstrou que existem mais questionamentos do que conclusões sobre as relações entre tutores e as ferramentas tecnológicas utilizadas nos cursos de capacitação de tutores. Por isso é possível dizer que esse trabalho contribui para implicações acadêmicas, no sentido de despertar discussões sobre o atual contexto histórico, no qual o conhecimento é mediado por instrumentos virtuais que transformaram completamente a perspectiva de tempo e espaço nos processos de ensinoaprendizagem.

Ressalta-se que o estudo tem limitações relacionadas ao seu próprio caráter qualitativo e ao baixo número de entrevistados, o que impede de estender as conclusões para a generalidade dos cursos para tutores ministrados por outras instituições.

Portanto, compreende-se que essa pesquisa se debruça sobre as relações do tutor com as ferramentas de trabalho em um curso de $\mathrm{EaD}$. Isso quer dizer que esse objeto é o ponto de encontro mediador entre o aluno do curso, o conteúdo e seus recursos materiais e pedagógicos. Dessa maneira, o processo de aprendizagem está condicionado à forma os tutores dominam essas ferramentas e o seu conteúdo, como também, às principais crenças sobre a EaD.

\section{REFERÊNCIAS}

ABREU, A. F.; GONÇALVEZ, C. M.; PAGNOZZI, L. Tecnologia da informação e educação corporativa: contribuições e desafios da modalidade de ensino-aprendizagem à distância no desenvolvimento de pessoas. Revista PEC, Curitiba, v. 3, n.1, p. 47-58, jul. 2002-jul. 2003.

\section{ALENCAR, E. Introdução à metodologia de} pesquisa. Lavras: UFLA, 2000. 105 p.

ALIPOUR, F.; IDRIS, K.; KARIMI, R. Knowledge Creation and Transfer: Role of 
Learning Organization. International

Journal of Business Administration, v. 2, n. 3, 2011 .

AZEVEDO, P.; BERNARDINI, M. S.; ROSA, R.; TERRA, L.; COSTA, A.;

DALMAU, M. Tics e a Aprendizagem

Continuada: O Caso do Curso de

Especialização em Gestão por Resultados,

Produtividade e Inovação da Parceria

Universidade Federal de Santa Catarina e

Ministério da Saúde. In: ESUD 2011 - VIII

Congresso Brasileiro de Ensino Superior a

Distância, Ouro Preto, out. 2011.

BANDEIRA-DE-MELLO, R.; CUNHA, C. J. C. A. Operacionalizando o Método da Grounded Theory nas Pesquisas em Estratégia: Técnicas e Procedimentos de Análise com Apoio do Software Atlas/TI. In: XXVII ENCONTRO ANUAL DA ASSOCIAÇÃO DOS PROGRAMAS DE PÓS-GRADUAÇÃO EM

ADMINISTRAÇÃO, Rio de Janeiro. Anais ANPAD, 2003.

BARDIN, L. Análise de conteúdo. Lisboa. ed. $70,1995.225 \mathrm{p}$.

BARRETO, R.G. Tecnologias na formação de professores: o discurso do MEC.

Educação e Pesquisa, São Paulo, v. 29, n. 2, p. 271-286, jul./dez. 2003.

BORBA, M. C.; MALHEIROS, A. P. S.; ZULLATO, R. B. A. Educação a distancia online. 2. ed. Belo Horizonte. Autentica, 2008.

BRUNI, A. L. Estatística aplicada à gestão empresarial. $2^{a}$ ed. São Paulo: Atlas, 2010.

COELHO, A. A.; SILVA, K. R. D.; TORRES, M. R. C.; FREITAS, L. B.; SERRA, M. R. S. A educação à distância como um desafio para os professores orientadores da especialização em educação do campo no município de São João dos Patos/MA. In: ESUD 2011 - VIII
Congresso Brasileiro de Ensino Superior a

Distância, Ouro Preto, out. 2011.

COOPER, D. R. ; SCHINDLER, P. S.

Métodos de pesquisa em administração. 7 . ed. Porto Alegre: Bookman. 2003. 640p.

FINCH, D.; JACOBS, K. Online Education: Best Practices to Promote Learning. Proceedings of the Human Factors and Ergonomics Society Annual Meeting. 56th. 2012.

MACHADO, F. H.; STANO, R. C. M. T.; LOTT, H. P.; JUNQUIEIRA, A. P.; OLIVEIRA, P. K. S. Ambientes virtuais de aprendizagem como espaço de afetividade: as interações no aprender. In: ESUD 2011 VIII Congresso Brasileiro de Ensino Superior a Distância, Ouro Preto, out. 2011.

MALHOTRA, N. Pesquisa de marketing: uma orientação aplicada. $4^{\mathrm{a}} \mathrm{ed}$. Porto Alegre: Bookman, 2006.

MAREK, K. Learning to Teach Online: Creating a Culture of Support for Faculty. Journal of Education for Library and Information Science, v. 50, n. 4, 2009.

MENDONÇA, R. R. S.; SANÁBIO, M. T.; CASTANHA, A. L. B.; FERRUGINI, L. Graduação em Administração a Distância: relatos, impressões e percepções sobre uma experiência pedagógica e social de valor agregado. In: ESUD 2011 - VIII Congresso Brasileiro de Ensino Superior a Distância, Ouro Preto, out. 2011.

NOGUEIRA, L. L. Educação a Distância. Comunicação e Educação. São Paulo, Moderna, ano II, n. 5, p. 34-9, jan./abr., 1996.

OLIVEIRA, A. S. Avaliação de Cursos de Pós-Graduação Lato Sensu a Distância. 2010.93 p. Dissertação (Mestrado em Administração). Universidade Federal de Lavras, Lavras, 2010. 
POMPEU, R. M.; SILVA FILHO, J. B. Uma experiência em EAD de cursos de Extensão via Internet em um Grupo Empresarial. Universidade de Fortaleza-UNIFOR, 2005.

PRETI, O. (org.) Educação a Distância: construindo significados. Cuiabá: NEAD: IE - UFMT; Brasília: Plano, 2000.

PRETTO, N. D. L.; RICCIO, N. C. R. A formação continuada de professores universitários e as tecnologias digitais. Educar em Revista. p. 153-169, 2010

RIENTIES, B.; BROUWER, N.; LYGOBAKER, $S$. The effects of online professional development on higher education teachers' beliefs and intentions towards learning facilitation and technology. Teaching and Teacher Education. v. 29, p. $122-131.2013$.

SENO, W. P.; BELHOT, R. V. Delimitando a fronteira para a identificação de competências para a capacitação de professores de engenharia para o ensino a distância. Gestão \& Produção, v. 16. p. 502514, 2009.

VERGARA, S. C. Métodos de Pesquisa em Administração. São Paulo: Atlas, 2005. 